\title{
Historical Aspects of Hyponatremia
}

\author{
Chris J. Thompson ${ }^{\mathrm{a}}$ Alessandro Perib ${ }^{\text {- Joseph G. Verbalis }}{ }^{\mathrm{c}}$

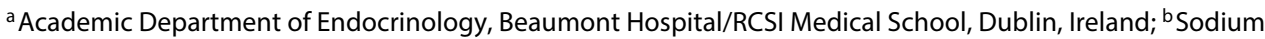 \\ Unit, Endocrinology, Department of Experimental and Clinical Biomedical Sciences "Mario Serio", "Center for \\ Research, Transfer and High Education on Chronic, Inflammatory, Degenerative and Neoplastic Disorders for \\ the Development of Novel Therapies" (DENOThe), University of Florence, Careggi University Hospital, Florence, \\ Italy; ' Division of Endocrinology and Metabolism, Georgetown University, Washington, DC, USA
}

In almost 2 decades since the onset of the 21 st century, there has been an explosion in interest in hyponatremia, which has resulted in a marked increase in the number of publications on the topic. This is remarkable as there is no evidence that the incidence or prevalence of the condition is increasing. Unlike new diseases, such as antibioticresistant infections or complications of the effects of cancer therapy, hyponatremia has always been there. However, the increased interest in hyponatremia reflects an improved understanding of the effects of hyponatremia on both morbidity and mortality, and equally, a recognition of the large gaps in our knowledge of this area. The expansion in the potential ramifications of hyponatremia, on falls, fractures and osteoporosis, as well as its association with increased mortality has focused on how much more still needs to be done to prove the causal relationships and to ascertain the value of therapeutic intervention. This has resulted in an academic environment characterised by healthy debate - and occasional dispute - among groups involved in hyponatremia research, which is beneficial since it further invigorates interest in hyponatremia. As a result, there has been a steady rise in publications on the topic of hyponatremia since the 1940s (Fig. 1).

However, the current clinical and academic interests in hyponatremia are relatively recent. For many years, hyponatremia was a topic that did not generate enthusiasm among journal editors or granting agencies, and indeed, was not attached to a specific sub-discipline of medicine. A small number of endocrinologists and nephrologists nurtured research programs that contributed to gathering knowledge on hyponatremia, but the majority of hospitals had no specialists who developed services for hyponatremic patients, or who set standards for investigations and management of the condition. The evolution of our knowledge on the physiology of the control of 


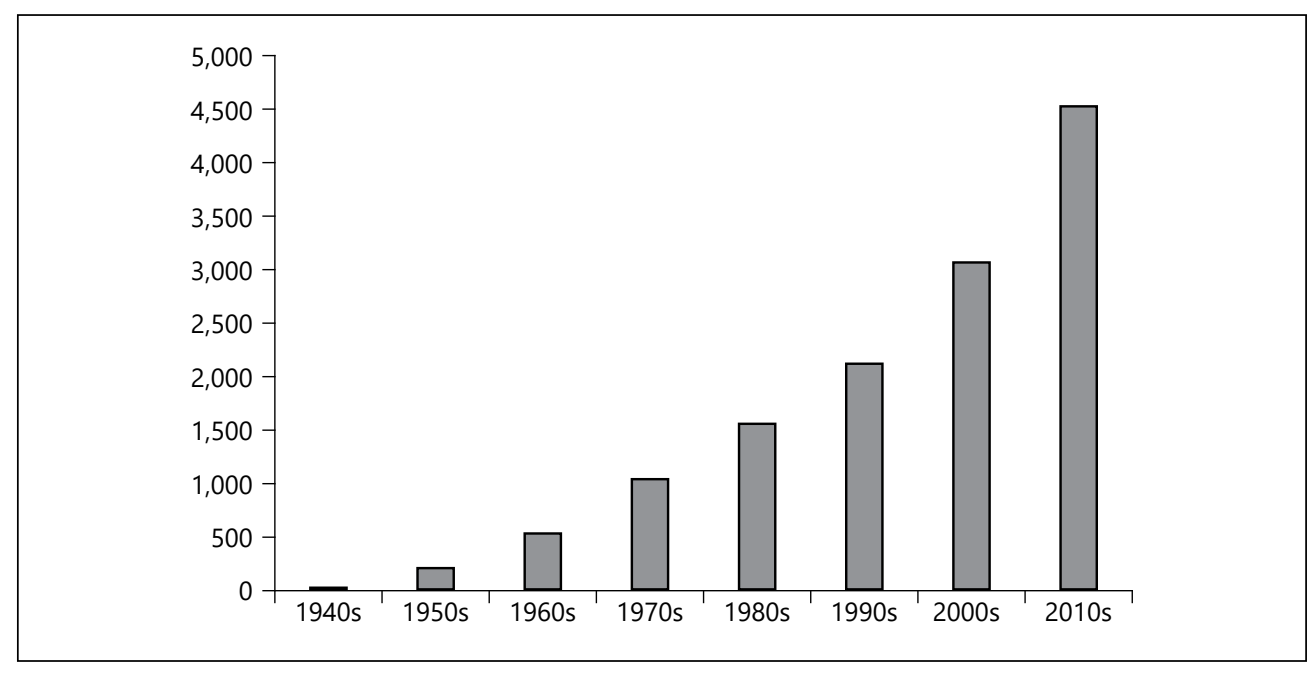

Fig. 1. Cumulative numbers of PubMed publications on hyponatremia, by decade.

serum $\left[\mathrm{Na}^{+}\right]$concentration, and how that knowledge transformed the clinical approach to diagnosis and management of hyponatremia, is a journey worthy of some comment.

\section{Early Publications and Theories}

Although there have been a number of papers that referred to hyponatremia in the context of water intoxication, the first systematic review of conditions marked by abnormalities of water and salt balance in PubMed was published in 1947 in the British Medical Journal [1]. Marriott's treatise focused on the prevailing interpretation of the time, that perturbations in serum $\left[\mathrm{Na}^{+}\right]$concentration could be explained on the basis of altered dynamics of sodium balance; in other words, that abnormal sodium intake or excretion was responsible for changes in serum $\left[\mathrm{Na}^{+}\right]$concentration, including hyponatremia. This view, which predominated the field for many years, is in direct contrast to modern interpretations of the pathophysiology of hyponatremia, which dictate that abnormalities of water handling, as mediated by the antidiuretic actions of arginine vasopressin (AVP) are responsible for a large number, if not most cases of hyponatremia [2]. The lack of awareness of the important role of water retention in hyponatremia was reflected in the first case series of patients who had hyponatremia complicating pulmonary tuberculosis [3]. Published as an abstract of a presentation to the American Society for Clinical Investigation, this paper detailed the clinical characteristics of 10 patients with advanced pulmonary tuberculosis, complicated by malnutrition, in whom hyponatremia (plasma sodium 123-130 $\mathrm{mEq} / \mathrm{L})$ was associated with elevated urine $\left[\mathrm{Na}^{+}\right]$concentrations. The clinical obser- 
vations indicated that there was no "frank evidence" of renal or adrenal disease, though two patients did test positive for adrenal insufficiency, using the archaic Levy-Power-Kepler test. The authors documented renal retention of sodium in 4 patients who were administered desoxycorticosterone, which raises the possibility that some of the patients were aldosterone deficient, perhaps due to tuberculous adrenalitis; 2 patients had recovery of serum $\left[\mathrm{Na}^{+}\right]$with improvement of their clinical condition, which suggests the possibility of the syndrome of inappropriate antidiuresis (SIAD) rather than adrenal failure. The authors concluded that the underlying pathophysiology was due to the "primary reduction in intracellular osmolality," and hypothesized that the increased renal sodium excretion reflected a homeostatic attempt to reduce extracellular tonicity to equilibrate with the reduced intracellular osmolarity. It is likely, without the full diagnostic criteria that we would consider essential today, that the case series was heterogeneous, with some patients having tuberculous adrenal destruction, and perhaps more having straightforward SIAD. However, despite the fastidious clinical documentation of the series, there was no stated awareness of the possibility of altered water excretion, either due to cortisol deficiency or excess AVP secretion, which are far likelier candidate pathophysiologies based on our current understanding of hyponatremia.

In subsequent years, the association of hyponatremia with hepatic cirrhosis [4] and congestive cardiac failure [5] were formally documented, but a key step forward came with the first description of what we now understand as the commonest cause of hyponatremia, SIAD.

\section{Early Descriptions of SIAD}

A critical advancement in our understanding of the pathogenesis of hyponatremia came in 1957 as a result of a series of clinical observations of 2 patients with the diagnosis of hyponatremia in the setting of bronchogenic carcinoma [6]. During the course of hospital admission, serum $\left[\mathrm{Na}^{+}\right]$fell significantly in both patients, to nadirs of 103 and $105 \mathrm{mEq} / \mathrm{L}$, respectively. In the first case, it was found that ad libitum salt intake and slightly restricted fluid intake improved hyponatremia, maintaining near normal plasma sodium concentration. In the second case, however, hyponatremia persisted, despite the use of large amounts of salt, and was accompanied by a continuous loss of sodium in the urine. There were no signs of extracellular fluid sodium or fluid depletion, and both patients revealed normal kidney, thyroid and adrenal functions. The urine remained continuously hypertonic compared to the plasma as serum $\left[\mathrm{Na}^{+}\right]$fell. Transient elevations of serum $\left[\mathrm{Na}^{+}\right]$were found with large loads of hypertonic saline infusion followed by a significant diuresis of dilute urine. Fluid restriction in both subjects resulted in sodium retention and a return of serum $\left[\mathrm{Na}^{+}\right]$to near normal values. Conversely, restoration of free fluid intake worsened the "sodiumlosing syndrome." 
The authors advanced the hypothesis that inappropriate urine concentration occurred in response to an antidiuretic hormone circulating in the plasma. Although they had no specific assay to measure plasma AVP concentrations, Schwartz et al. [6] were able to define the main principles of the diagnosis and treatment of this condition, such that their seminal paper remains the gold standard description of SIAD today. Interestingly, the fluid restriction that they used as a treatment for hyponatremia continued to be endorsed for years [7], and is still recommended by recent international guidelines as the first-line treatment for mildly symptomatic SIAD [8].

\section{Measurement of Plasma Vasopressin Levels}

The ability to measure plasma concentrations of AVP was a major advancement, as this allowed definition of the characteristics of the regulation of secretion of the antidiuretic hormone for the first time $[10,11]$. Prior to the development of sensitive radioimmunoassays for the measurement of AVP in plasma, our knowledge of the physiology of AVP release and action was implied from the use of urine osmolality as a bioassay for the action of AVP. In the 1970s, a number of specific antibodies to AVP were developed from animal models, and used to develop sensitive radioimmunoassays, which were used in clinical studies to measure AVP in plasma and urine [9]. Using these new assays, researchers defined the physiology of AVP secretion in response to osmotic and non-osmotic stimuli [12]. With particular relevance to hyponatremia, Robertson et al. [13] were able to demonstrate that plasma AVP concentrations were elevated in $>90 \%$ of cases of SIAD [13], thus proving the clinical hypothesis made by Schwartz et al. [6] 25 years earlier.

\section{Development of Animal Models of Hyponatremia}

Although the availability of radioimmunoassays allowed the characterization of the physiological control of AVP secretion and the relationship of abnormal osmoregulation to the development of hyponatremia, the establishment of animal models of hyponatremia allowed more complex pathophysiological issues to be explored. The neurological sequelae and high mortality of acute and chronic hyponatremia did not readily allow clinical research protocols in humans, which suggested the need for translational studies in animal models.

The first published description of symptoms of water intoxication in humans was reported in 1923 [14], followed by studies in experimental animals that showed cerebral edema as the likely cause of neurological symptoms [15]. Not much later, the first case of fatal cerebral edema was reported in a post-operative patient [16], which was quickly followed just 3 years later by a report of the first successful treatment of hyponatremic encephalopathy using hypertonic $\mathrm{NaCl}$ [17], a remarkable example of 
rapid translation from basic studies in animals to an effective clinical therapy that is still used today.

Over subsequent years, many studies were carried out in animals using protocols employing administration of AVP in conjunction with water loading, usually by intraperitoneal administration of $5 \%$ dextrose solution. Although important discoveries were made, notably the first description of brain electrolyte losses during adaptation to hyponatremia [18], all of these early models were accompanied by high rates of morbidity and mortality, making studies of chronic hyponatremia difficult to perform and even more challenging to interpret. A more stable rat model of SIAD was developed in the US using desmopressin-induced antidiuresis in combination with water loading via self-ingestion of a liquid diet that had negligible effects on mortality and proved to be a good model for human osmoregulation [19], which then allowed studies of the abnormal osmoregulation during sustained hyponatremia [20]. Having established credentials as a valid animal model for human SIAD, a series of studies was then able to establish the patterns of osmolyte fluxes across the brain and the abnormalities that were present during hyponatremia [21]; the data from these studies were also used to develop a model to explore the abnormal osmolyte fluxes after overly rapid correction of hyponatremia [22]. The knowledge gained from the use of an experimental animal model of SIAD has provided the basis for much of what we understand about the pathophysiology of osmotic demyelination, the dreaded sequel of overly rapid correction of chronic hyponatremia [23], which can lead to spastic paraplegia and cranial nerve palsies. The knowledge gained from these translational studies complemented observational studies in human patients [27], which in combination directly led to the guidelines for the treatment of hyponatremia that reduced the risk of osmotic demyelination [8].

\section{Recent Advances}

The identification of the aquaporin water channels, particularly the AVP-regulated collecting duct aquaporin-2 channel, has significantly elevated our understanding of the actions of AVP in the reabsorption of water from the renal tubules [24]. This fundamental discovery was a prime example of the ability of translational basic science studies to enable clinicians to better understand body physiology and pathophysiology at a clinically relevant level. Many of the inherited mutations and acquired metabolic malfunctions of the aquaporins produce polyuria rather than hyponatremia. However, the unusual mutation responsible for the nephrogenic syndrome of antidiuresis [25] has been shown to cause hyponatremia despite undetectable plasma concentrations of AVP, because of a gain-of-function mutation of the AVP V2 receptor, leading to the recruitment of aquaporin-2 in large numbers to the apical membrane of collecting duct principal cells resulting in AVP-independent constitutive antidiuresis. 
The discovery of the AVP receptor antagonists (vaptans) has also been a major advancement in the therapeutic approach to hyponatremia, with an emphasis on SIAD. The indications and therapeutic advantages of this class of drugs will be covered in depth in the relevant chapters on SIAD, but it is clear from existing randomized clinical trials that they are effective for the treatment of hyponatremia, and clinical experience has shown few serious adverse reactions. A recent review surmised that reticence about more widespread use are based partly on the economics of therapy and partly on a lack of hard end-point data [26]. The need for better prospective data to inform our diagnostic and therapeutic decisions in hyponatremic patients will be a recurrent theme in this book.

It gives us great pleasure as editors to introduce this volume of work on the subject of hyponatremia. For each of us, our study and practice of endocrinology has been dominated by an interest in this fascinating subject. It has been a pleasure to witness the upsurge in interest and publications on hyponatremia over the last 2 decades, as clearly illustrated in Figure 1. The chapters in this book have been selected by editors to update key areas of recent research in hyponatremia that have fuelled the recent rise in publications. To do this, we have gathered a team of truly expert enthusiasts to cover these important topics. We hope that the volume will be a useful and important summary of our evolving understanding of hyponatremia, and will stimulate further research into the still large number of questions that remain to be answered about this important disorder.

Even a cursory glance at Figure 1 is sufficient to comprehend the steady increase in research on hyponatremia, with well over 4,000 publications up to the end of 2017. The editors fully expect that this trend will not only continue, but will accelerate with the increasing volume of basic, translational and clinical studies in sodium and water metabolism.

\section{References}

1 Marriott HL: Water and salt depletion. BMJ 1947;1: 328-332.

2 Sterns RH, Silver SM: Complications and management of hyponatremia Curr Opin Nephrol Hypertens 2016;25:114-119.

3 Sims EA, Welt LG, Orloff J, Needham JW: Asymptomatic hyponatremia in pulmonary tuberculosis. J Clin Invest 1950;29:846-847.

4 Nelson WP 3rd, Rosenbaum JD, Strauss MB: Hyponatremia in hepatic cirrhosis following paracentesis. J Clin Invest 1951;30:738-744.

5 Elkinton JR, Squires RD: The therapeutic significance of hyponatremia in congestive heart failure. Trans Stud Coll Physicians Phila 1951;18:145.
6 Schwartz WB, Bennett W, Curelop S, Bartter FC: A syndrome of renal sodium loss and hyponatremia probably resulting from inappropriate secretion of antidiuretic hormone. Am J Med 1957;23:529-542.

7 Schrier RW: Treatment of hyponatremia. N Engl J Med 1985;312:1121-1123.

8 Verbalis JG, Goldsmith SR, Greenberg A, Cornelius C, Schrier RW, Sterns RH, Thompson CJ: Diagnosis, evaluation, and treatment of hyponatremia: expert panel recommendations. Am J Med 2013;126(10 suppl 1):S1-S42.

9 Robertson GL, Mahr EA, Athar S, Sinha T: Development and clinical application of a new method for the radioimmunoassay of arginine vasopressin in human plasma. J Clin Invest 1973;52:2340-2352. 
10 Baylis PH, Heath DA: The development of a radioimmunoassay for the measurement of human plasma arginine vasopressin. Clin Endo 1977;7:91-102.

11 Rooke P, Baylis PH: A new sensitive radioimmunoassay for plasma arginine vasopressin. J Immunoassay 1982;3:115-131.

12 Robertson GL: The regulation of vasopressin function in health and disease. Recent Prog Horm Res 1976;33:333-385.

13 Robertson GL, Aycinena P, Zerbe RL: Neurogenic disorders of osmoregulation. Am J Med 1982;72: 339-353.

14 Rowntree LG: Water intoxication. Arch Int Med 1923;32:157-174.

15 Rowntree LG: The effects on mammals of the administration of excessive quantities of water. Pharmacol Exp Ther 1926;29:135.

16 Helwig FC: Water intoxication: report of a fatal human case, with clinical, pathologic and experimental studies. JAMA 1935;104:1569.

17 Helwig FC, et al: Water intoxication. Moribund patient cured by administration of hypertonic salt solution. JAMA 1938;110:644-645.

18 Yannet H: Changes in the brain resulting from depletion of extracellular electrolytes. Am J Physiol 1940;128:683-689.

19 Verbalis JG: An experimental model of syndrome of inappropriate antidiuretic hormone secretion in the rat. Am J Physiol 1984;247:E540-E553.
20 Verbalis JG, Baldwin EF, Robinson AG: Osmotic regulation of plasma vasopressin after sustained hyponatremia. Am J Physiol 1986;250:R444-R451.

21 Verbalis JG, Gullans SR: Hyponatremia causes large, sustained reductions in brain content of multiple organic osmolytes in rats. Brain Res 1991;20:274-282.

22 Verbalis JG, Gullans SR: Rapid correction of hyponatremia produces differential effects on brain osmolytes and electrolyte accumulation in rats. Brain Res 1993;19:19-27.

23 Verbalis JG: Control of brain volume during hypoosmolality and hyperosmolality. Adv Exp Med Biol 2006;576:113-129.

24 Agre P: Aquaporin water channels in kidney. J Am Soc Nephrol 2000;11:764-777.

25 Feldman BJ, Rosenthal SM, Vargas GA, Fenwick RG, Huang EA, Matsuda-Abedini M, Lustig RH, Mathias RS, Portale AA, Miller WL, Gitelman SE: Nephrogenic syndrome of inappropriate antidiuresis. $\mathrm{N}$ Engl J Med 2005;352:1884-1890.

26 Rondon-Berrios H, Berl T: Vasopressin receptor antagonists in hyponatremia: uses and misuses. Front Med (Lausanne) 2017;4:141.

27 Sterns RH, Riggs JE, Schochet SS Jr.: Osmotic demyelination syndrome following correction of hyponatremia. N Engl J Med 1986;314:1535-1542.

\author{
Alessandro Peri \\ Sodium Unit, Endocrinology \\ Department of Experimental and Clinical Biomedical Sciences "Mario Serio" \\ University of Florence, Viale Pieraccini, 6 \\ Careggi University Hospital \\ IT-50139 Florence (Italy) \\ E-Mail alessandro.peri@unifi.it
}

